\title{
Imaging Properties and Aberration Analysis of Electrostatic Afocal-Zoom Lenses Using Computer Optimization
}

\section{Omer Sise}

\author{
Department, of Science Education, Faculty of Education, Suleyman Demirel University, 32260 Isparta, \\ Turkey.
}

The focusing properties of electrostatic lenses have been extensively studied both experimentally and theoretically due to their importance in practical applications in electron spectroscopy, surface science, electron microscopy, and mass spectrometry [1-3]. These applications require the optimization of the various lens parameters, such as a set of voltages applied to electrodes, to achieve minimum beam distortion, small aberrations and high transmission. It is well known that multi-element lenses are very useful in electron-optical systems due to their exceptional focusing capabilities. In most of the applications, it is suitable to vary the final-to-initial electron energy without altering the object and image positions, $P$ and $Q$, respectively [4]. This condition can be obtained by using three-element "zoom" lenses [1]. Although the two voltage ratios are varied to keep the object and image positions constant as the overall voltage ratio is varied, the linear and/or angular magnification, $M$ and $M_{\alpha}$, will change. Figure 1a shows schematically the focusing and the magnification of a zoom lens. In general, to keep $n$ imaging properties constant while varying final-to-initial electron energy, a lens system with $n+2$ electrodes is needed. If one wants to control the image position together with one of the magnifications $\left(M\right.$ or $M_{\alpha}$ ) for a given object position, an extra element must be introduced [5]. Lenses with more than four elements can have other advantages, such as lower aberration, a more extended range of overall voltage ratio or special lens modes like afocal lenses [6,7], i.e., parallel rays entering the lens will exit parallel, and the beam angle $\left(\theta_{b}\right)$ is zero. Here, the beam angle is a vital parameter to improve the angular spread of the beam, which is particularly important for high-resolution electron spectrometers [8-11]. This angle can be controlled well if the potentials are varied so as to make the beam angle zero at the image side (the beam is collimated). So, in order to control independently i) the image position $Q$, ii) the magnification $M$, and iii) the beam angle $\theta_{b}$ while the final-to-initial electron energy is varied, a minimally five-element lens system is necessary. Operating the lens in "afocal-zoom" mode, i.e., a parallel input beam with respect to the optical axis leaves the lens parallel to the axis, and the beam at the object position is focused to the image position, as shown in Figure 1b, would be helpful to reduce the angular divergence of the beam prior to the electrostatic energy analyzers (see, for example, Ref. [12]).

An electrostatic lens transports the electrons emanated from the source to the image point of the lens with certain acceleration/deceleration and linear magnification $M$. As it is well known, the following relation holds for imaging using such a lens system

$$
r_{o} \alpha_{o} V_{1}^{1 / 2}=r_{i} \alpha_{i} V_{n}^{1 / 2} \quad \text { (the Liouville theorem) }
$$

where $\alpha_{i}$ and $\alpha_{o}$ are the pencil angles of the electrons at the image and the object position, respectively, and $r_{i}$ and $r_{o}$ are the image and the original object sizes. The quantity $V_{n} / V_{1}$ defines the acceleration/deceleration ratio, where the $V_{1}$ and $V_{n}$ are the potentials at the first and last electrode, respectively. As shown in Figure 1a, a particle starts at the object plane, off-axis, with direction parallel to the axis. The vertical position at which it crosses the image plane determines the linear magnification, 
and the angle it crosses at is zero if lens is operated in afocal mode (Figure 1b). The other particle starts at the image plane, on-axis, with direction at a small pupil angle to the axis. The horizontal position and angle at which it crosses the axis determines the image plane.

\section{a) Zoom lens}

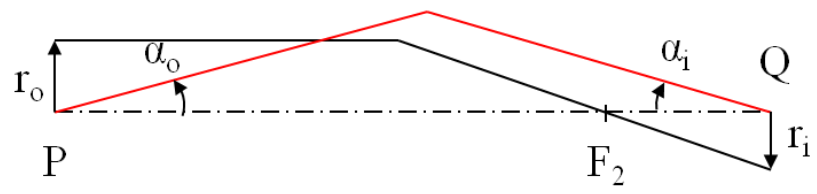

b) Afocal-zoom lens

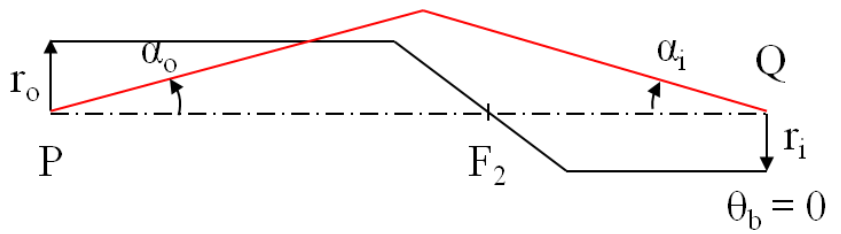

Figure 1. Different modes of electrostatic lens usage. The object and image positions, $P$ and $Q$, are defined as crossing points on the lens axis. (a) illustrates the focusing of both on- and off-axis rays in the lens, but the beam angle at the image side is not zero. The condition satisfied here is that an object point at $P$ should be focused at $Q$. The 2nd parallel ray is only used to measure the magnification $M$ at $Q$. There is no condition on $M$ or the focal point $F_{2}$. (b) demonstrates the afocal-zoom lens. A parallel input beam with respect to the optical axis leaves the lens parallel to the axis, and the beam at the object position is focused to the image position. Here, there are two constraints: $i)$ keep the beam angle zero $\left(\theta_{\mathrm{b}}\right.$ $=0$ ) and ii) keep the image point at $Q$. Note that the bundle size or magnification may change, depending on the position of $F_{2}$.

The final radius of the beam is given by the summation of the different contributions,

$$
r_{f}^{2}=\left(M r_{o}\right)^{2}+\left(\Delta r_{i} / 4\right)^{2}+\left(\delta r_{i}\right)^{2}
$$

Here, $\Delta r_{i} / 4$ is the radius of the disc of least confusion due to spherical aberration, defined as

$$
\Delta r_{i}=M C_{s o} \alpha_{o}^{3}
$$

where $C_{s o}$ is the object-side spherical aberration coefficient, and the contribution from the chromatic aberration is given by

$$
\delta r_{i}=M C_{c o} \alpha \Delta T / T
$$

where $C_{c o}$ is the object-side chromatic aberration coefficient, $T$ is the beam energy and $\Delta T / T$ resembles the relative energy spread. Here, the object side aberrations $\left(C_{s o}\right.$ and $\left.C_{\mathrm{co}}\right)$ image side aberration $\left(C_{s i}\right.$ and $\left.C_{\mathrm{ci}}\right)$ are connected by $C_{s o}=\left(C_{s i} / M^{4}\right)\left(V_{1} / V_{n}\right)^{3 / 2}$ and $C_{\mathrm{co}}=\left(C_{\mathrm{ci}} / M^{2}\right)\left(V_{1} / V_{\mathrm{n}}\right)^{3 / 2}$.

With this in mind, the present work targets the optimization of the voltage settings of a five-element electrostatic afocal-zoom lens as a function of the overall voltage ratio by simplex optimization. In a previous paper [13], the voltage ratios of $V_{2} / V_{1}, V_{3} / V_{1}, V_{4} / V_{1}$, and the corresponding aberration coefficients (spherical $C_{s o}$ and chromatic $C_{c o}$ ) were analyzed with a range of linear magnification $(M)$ of between 0.3 and 3.2 for selected final to initial voltage ratios $\left(V_{5} / V_{1}=1 / 4,1,4\right)$. In the present paper we extend our analysis to a full range of final-to-initial voltage ratios of between 0.03 and 33 (a total span of $\left.1: 10^{3}\right)$. 
The five-element lens is simple to design, yet the electron-optical properties of the lens are quite complex due to the large number of degrees of freedom in the lens. For minimization of complicated non-linear functions, the application of simplex optimization methods was found to be very effective (see Ref. [13], where the ray tracing method employed was described). We extensively utilize the charged particle simulation program SIMION v8.1 [14] in the optimization phase. It produces the particle tracking on 3D electrostatic potential arrays determined by solving the Laplace equation, integrating the equations of motion in the electrostatic field using the standard Runga-Kutta method. In Figure 2, we have simulated the ray trajectories for the symmetric case of the five-element lens with cylinder lengths of $L_{1}=L_{5}=1.5, L_{2}=L_{4}=1.0$, and $L_{5}=3.0$ diameters, $D$. In numerical calculations, the gaps between the cylinders are $G=0.1 D$, and the potential of the first electrode is $V_{1}=1 \mathrm{~V}$. The positions of object and image distances are fixed at $P=Q=4 D$. Specifically, electrons were used to demonstrate the imaging properties of the lens. Inverting the voltage settings on the electrodes will permit imaging of positive ions in a similar way.

For a given $V_{5} / V_{1}$, the potentials on the three inner electrodes are adjustable parameters for obtaining defined linear magnification for a fixed object and image distances. By simultaneously adjusting $V_{2} / V_{1}$, $V_{3} / V_{1}$, and $V_{4} / V_{1}$ as $V_{5} / V_{1}$ is varied, the conditions for afocal-zoom lenses can be satisfied. The simplex optimization is used to optimize voltages for desired linear and angular magnifications. We compute a single metric for optimization routine to minimize. The metric is taken as the sum of five other individual metrics representing parameters we want to minimize. Each individual metric is defined to be non-negative and scaled so that the lens parameters $\left(M, V_{2}, V_{3}, V_{4}\right)$ are recorded if the total metric is less than 1. Figure 2a illustrates the focusing of both on- and off-axis rays in the five-element lens with two optimized voltage ratios $V_{2}$ and $V_{4}$, but the beam angle at the image side is not zero. To make the beam angle zero, the third voltage $V_{3}$ is optimized such that parallel rays entering the lens will exit parallel, although the bundle size or magnification may change. Figure $2 \mathrm{~b}$ demonstrates the focusing capability of the afocal-zoom lens.
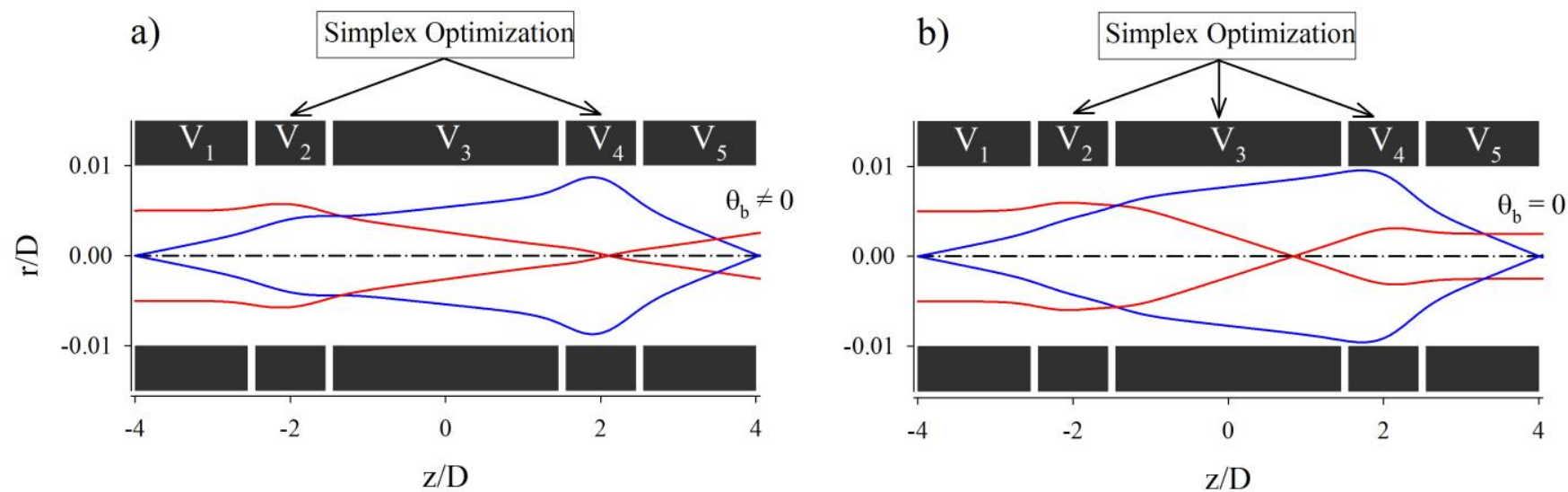

Figure 2. Simulated electron trajectories through the five-element lens. The object and image positions, $P$ and $Q$, are defined as crossing points with the lens axis. For all cases considered $P=4 D$ and $Q=4 D$ are fixed, so the total distance is $8 D$. It is clearly shown in (b) that the beam angle is controlled well if the potential of the centre electrode $\mathrm{V}_{3}$ is varied so as to make the beam angle zero at the image side (the beam is collimated). The trajectories are expanded in vertical direction for clarity. 
There are many possible sets of voltage ratios for $M=1$ in different modes of operation. The afocal fiveelectrode lens of Heddle [7] has a voltage combination of $V_{3}<V_{2}$ and $V_{3}<V_{4}$ for $V_{5}=V_{1}$ mode 4 in Figure 3. We found that other voltage combinations are also possible: mode $1: V_{3}>V_{2}, V_{3}>V_{4}$; mode 2: $V_{3}>V_{2}, V_{3}<V_{4}$; mode 3: $V_{3}<V_{2}, V_{3}>V_{4}$. In Figure 3, the dependence of voltage ratios $V_{2} / V_{1}, V_{3} / V_{1}$ and $V_{4} / V_{1}$ on the overall voltage ratio $V_{5} / V_{1}$ with a unit beam magnification is illustrated numerically for the afocal-zoom lens. A critical issue for such lenses is the minimization of aberrations. So, the aberration coefficients $C_{s o} / D$ and $C_{c o} / D$ are also given in Figure 4. The simulations of different modes of afocalzoom lens demonstrate that mode 1 produces much lower aberration coefficients than other modes.
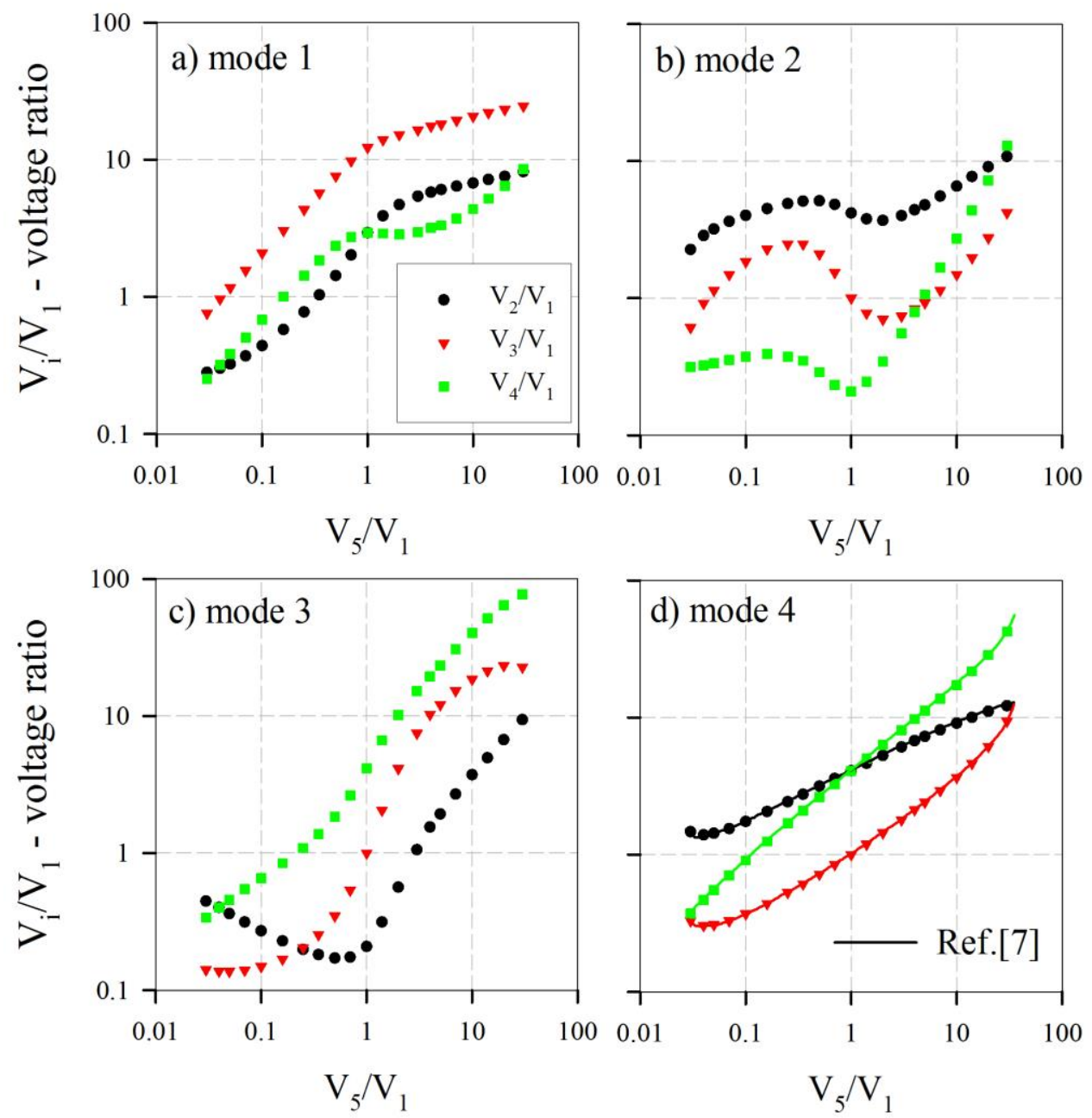

Figure 3. Dependence of voltage ratios of afocal-zoom lens on the overall voltage ratio for a linear magnification of 1. The solid lines in (d) denote the calculation of Heddle and Kay [7]. 

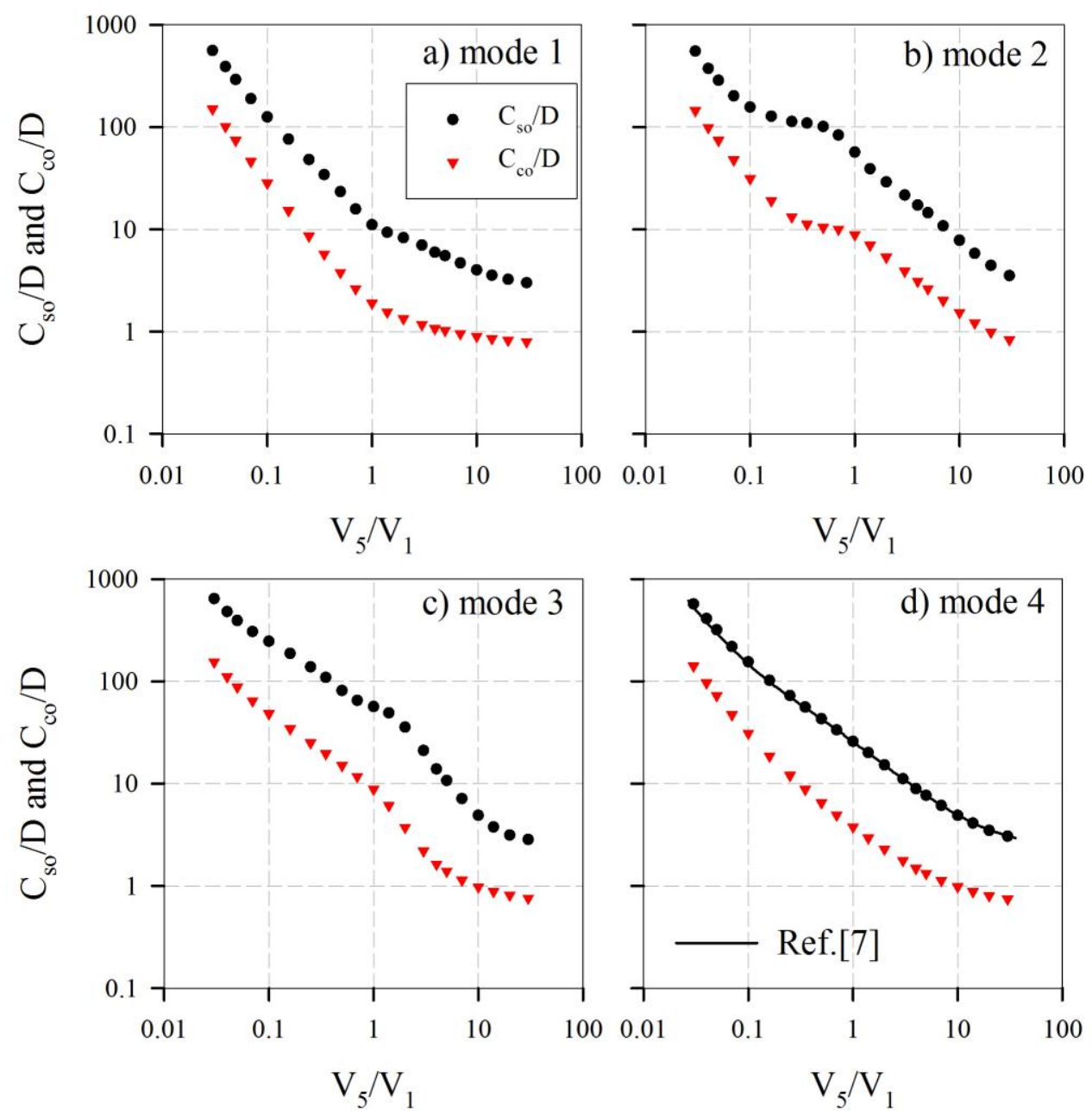

Figure 4. Dependence of aberration coefficients of afocal-zoom lens on the overall voltage ratio for a linear magnification of 1 . The solid line in (d) denotes the calculation of Heddle and Kay [7].

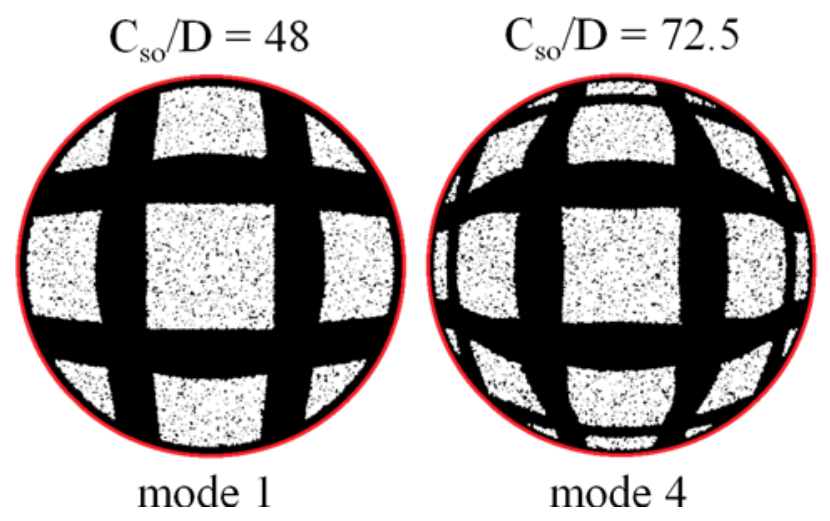

Figure 5. Comparison of the shadow-graphs of two modes (mode 1 and mode 4) for $V_{5} / V_{1}=0.25$ and $M$ $=1$. 
We have also used the grid-shadow method to show the effect of the spherical aberrations on image formation. A high resolution square grid with a spacing of $12.5 \mu \mathrm{m}$ and a thickness of $4 \mu \mathrm{m}$ was modelled in 3D using a SIMION geometry file. The electron trajectories from the object position $P$ passes through the lens, makes an image at $Q$, passes through the grid situated just after the Gaussian image plane, and casts a shadow upon a screen. The simulation results for the two modes in comparison are shown in Figure 5 for the lens with $V_{5} / V_{1}=0.25$ and $M=1$. It is clearly seen that the curvature of the shadows increases as the spherical aberration coefficient $C_{s o}$ increases.

In summary, we have found a full range of voltage ratios and aberration coefficients for a five-element cylindrical electrostatic lens operated in afocal-zoom mode with a range of final-to-initial voltage ratios $\left(V_{5} / V_{1}\right)$ of between 0.03 and 33 (a total span of $1: 10^{3}$ ) using a computer optimization technique. A major advantage of the afocal-zoom lens is that the magnification can be tuned to any desired value with zero beam angle by adjusting the voltages that are applied to the central electrodes. Although this article presents an optimization method for producing such an afocal-zoom lens, it can be applied to various problems in charged particle optics such as fringing field correction of electrostatic energy analyzers, high demagnification focused ion beam columns, and etc. Design of electrostatic lenses is still popular in charged particle optics. In the simulation presented here and in Ref. [13], an idea has been given in regard to optimizing the multi-element lens system.

Geometric lens optimization is also critical for achieving small aberrations. We are currently exploring a variety of geometries that could optimize these parameters using genetic algorithm [15].

\section{References:}

[1] E Harting and FH Read in "Electrostatic Lenses", (Elsevier, Amsterdam, 1976).

[2] B Lencova in "Handbook of Charged Particle Optics", ed. J Orloff, (CRC Press, Boca Raton) (2008), p. 161.

[3] M Yavor, Adv. Electron. El. Phys. 157 (2009), p. 213.

[4] PW Hawkes and E Kasper in "Principles of Electron Optics: Applied Geometrical Optics, vol. 2" (Academic Press, 1989), p.647.

[5] G Martinez, M Sancho and FH Read, J. Phys. E 16 (1983), p.631.

[6] DWO Heddle, J. Phys. E: Sci. Instrum. 4 (1971), p.981.

[7] DWO Heddle and SM Kay, Nucl. Instrum. Meth. A 298 (1990), p.291.

[8] T Sakae et al, Rev. Sci. Instrum. 61 (1990), p.61.

[9] R Bertacco et al, Rev. Sci. Instrum. 73 (2002), p.3867.

[10] TJM Zouros and EP Benis, Appl. Phys. Lett. 86 (2005), 094105.

[11] M Dogan et al, Rev. Sci. Instrum. 84 (2013), 043105.

[12] L Víkor et al, Nucl. Instrum. Meth. B 114 (1996), p.164.

[13] O Sise, J. Electron Spectrosc. 197 (2014), p. 7.

[14] SIMION 3D v8.1, Scientific Instrument Services Inc., www.simion.com.

[15] Mr. David Manura from Scientific Instrument Services (SIS) is thanked for his many useful discussions and providing technical support in SIMION simulation. 